\title{
Bending the parallels of the Poggendorff figure
}

\author{
MITCHELL BRIGELL \\ Northeastern University, Boston, Massachusetts 02115 \\ and \\ JOHN UHLARIK \\ Kansas State University, Manhattan, Kansas 66506
}

\begin{abstract}
The two angles formed by the intersection of the transversal segment and parallel line of the Poggendorff figure were independently varied in size by bending the parallel at the intersection. The standard illusion could be enhanced or reversed by manipulating the size of the obtuse $(\beta)$ angle. Regardless of acute $(\alpha)$ angle size, a maximal illusion obtained for $\alpha=120 \mathrm{deg}$, and the illusion was reversed for figures in which $\beta<\alpha$.
\end{abstract}

In the standard Poggendorff illusion, two segments of an oblique transverse line (labeled $x-y$ in Figure 1A) appear misaligned in the presence of two parallel vertical lines. Specifically, continuation of the lower segment of the transversal would appear to intersect the right parallel line below the vertex formed by the upper segment and the right vertical line. A traditional explanation of this effect involves the regression of acute and obtuse angles toward right angles. That is, acute angles are perceptually expanded, and obtuse angles are perceptually contracted. Recent research has documented that angles regress perceptually toward $90 \mathrm{deg}$ (Bouma \& Andriessen, 1970; Carpenter \& Blakemore, 1973; Hotopf \& Ollerearnshaw, 1972). However, empirical verification of the role of angle misperception in the Poggendorff illusion has been more difficult (Day \& Dickinson, 1975; Hotopf \& Olerearnshaw, 1972).

In the past, angle size in the Poggendorff figure has been manipulated by rotating the oblique segments around their vertices (Pressey \& Sweeney, 1972; Velinsky, 1925; Weintraub \& Krantz, 1971). This manipulation necessarily confounds the effects of acute and obtuse angle size in that any change in the size of angle $\alpha$ is necessarily accompanied by a complementary change in the size of angle $\beta$. Thus, showing that this manipulation affects the Poggendorff illusion is not sufficient information to assess the independent effects of the size of angles $\alpha$ and $\beta$ or to test angle-distortion theory. In the present series of experiments, the size of acute $(\alpha)$ and obtuse $(\beta)$ angles was manipulated independently in the Poggendorff figure by bending the parallels at the

Portions of this paper were presented at ARVO (Orlando, Florida, May 1980). We wish to express our appreciation to Rick Pringle for commenting on an earlier version of this report and to Judy Andrews and Kevin Jordan for their help in carrying out the experiments. Requests for reprints should be addressed to Mitchell Brigell, Department of Neurology, Michael Reese Hospital and Medical Center, 29th Street and Ellis Avenue, Chicago, Illinois 60616. vertices they make with the oblique lines (see Figure 1). By bending the parallels, the interaction between the effects of angles $\alpha$ and $\beta$ on the Poggendorff illusion can be assessed.

Predictions regarding effects of bending the parallels can be made using the data of Carpenter and Blakemore (1973). These authors measured the perceived orientation of an arm of an angle directly by having the observer set a third line parallel to it. Acute angles were overestimated and obtuse angles were underestimated in size. The largest effect, an overestimation of approximately 2 deg, occurred for an acute angle of $15 \mathrm{deg}$. A 90-deg angle was accurately perceived, and the largest underestimation of an obtuse angle (1 deg) occurred for

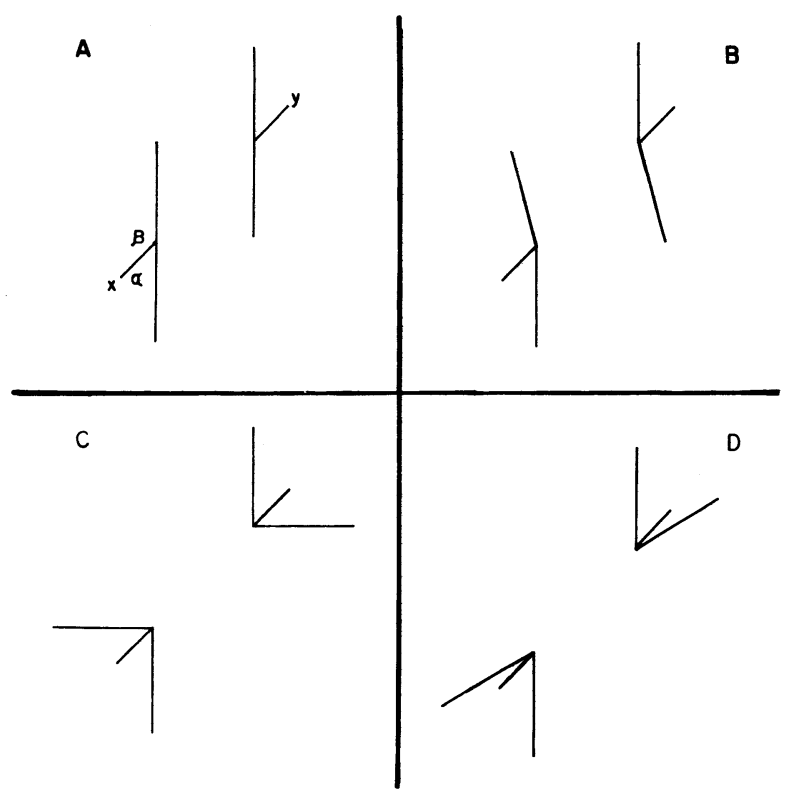

Figure 1. Examples of stimuli used in the present study. For all stimuli shown, $\alpha=45 \mathrm{deg}$. From $A$ to $D, \beta=135,120,45$, and $15 \mathrm{deg}$, respectively. 
angles of $120-135 \mathrm{deg}$. In applying these data to the Poggendorff illusion, the two angles formed by the parallel and transversal lines can be treated as vectors acting on the perceived orientation of the transversal. For example, in the standard Poggendorff figure, with straight parallels and an angle $\alpha$ of $45 \mathrm{deg}$, the acute angle would perceptually rotate the oblique $1 \mathrm{deg}$ toward horizontal ("expansion"), and the 135-deg angle $\beta$ would concurrently act on the precept of the transversal, rotating it toward the horizontal $1 \mathrm{deg}$ ("contraction" of the obtuse angle). Thus, the net effect of the parallel upon the perceived orientation of the oblique is a 2-deg rotation toward horizontal. As the size of $\beta$ is decreased by rotating both sections of the parallels forming the obtuse angle counterclockwise in 15-deg steps, the illusion should increase in magnitude until angle $\beta$ is $120 \mathrm{deg}$. This effect should diminish with further reductions in the size of $\beta$ until the illusion reverses, when $\beta$ is smaller than angle $\alpha$. Some of these Poggendorff figures with bent parallels are illustrated in Figures 1A-1D. The exaggeration and reversal of the standard illusion are apparent in these figures. A formal test of the vector model was carried out in the experiments to follow.

\section{EXPERIMENT 1}

The purpose of this experiment was to examine the effects of independently manipulating the size of angles $\alpha$ and $\beta$ to evaluate their effect on the appearance of the transversal.

\section{Method}

Twenty-four undergraduates enrolled in the general psychology course at Kansas State University participated in the experiment as part of a course requirement. Four subjects were run per session.

The stimuli were drawn in black ink on $22 \times 28 \mathrm{~cm}$ sheets of paper. The width of the lines was $.35 \mathrm{~cm}$. Each segment of the transversal was $3 \mathrm{~cm}$ long and bisected a $10-\mathrm{cm}$ parallel. The width between the parallels was $4.3 \mathrm{~cm}$ for all stimuli.

Each observer judged a total of 28 stimuli: 21 test figures, 6 anchor stimuli, and a control stimulus. Two test figures were standard Poggendorff figures with 30- and 45-deg $\alpha$ angles and straight parallels (i.e., $\beta$ angles of 150 and $135 \mathrm{deg}$, respectively), and two were variations of the Poggendorff in which there was no obtuse angle (i.e., $\beta=0$ ). The remaining figures had bent parallels. The bent-parallel figures had values of $\beta$ intermediate between the standard Poggendorff and the $\beta=0$ figures. Angle $\beta$ was incremented in 15-deg steps, resulting in eight figures with bent parallels for a $45-\mathrm{deg}$ angle $\alpha$ ( $\beta$ ranging in size from 15 to $120 \mathrm{deg}$ ) and nine figures with bent parallels for a 30-deg angle $\alpha$ ( $\beta$ ranging from 15 to $135 \mathrm{deg}$ ). The control stimulus, which had no parallels and therefore no angles, was included to determine the point of subjective equality (PSE), and six anchor stimuli, in which the transversal segments were physically displaced, were included to encourage the observers to use a wide range of the response scale. Stimuli were presented in two booklets, one containing all figures with $\alpha=30 \mathrm{deg}$ and the other containing all 45-deg angle $\alpha$ figures. Stimulus order was randomized within each booklet, with the exception of the anchor stimuli, which were placed in the first three and last three positions of each booklet.

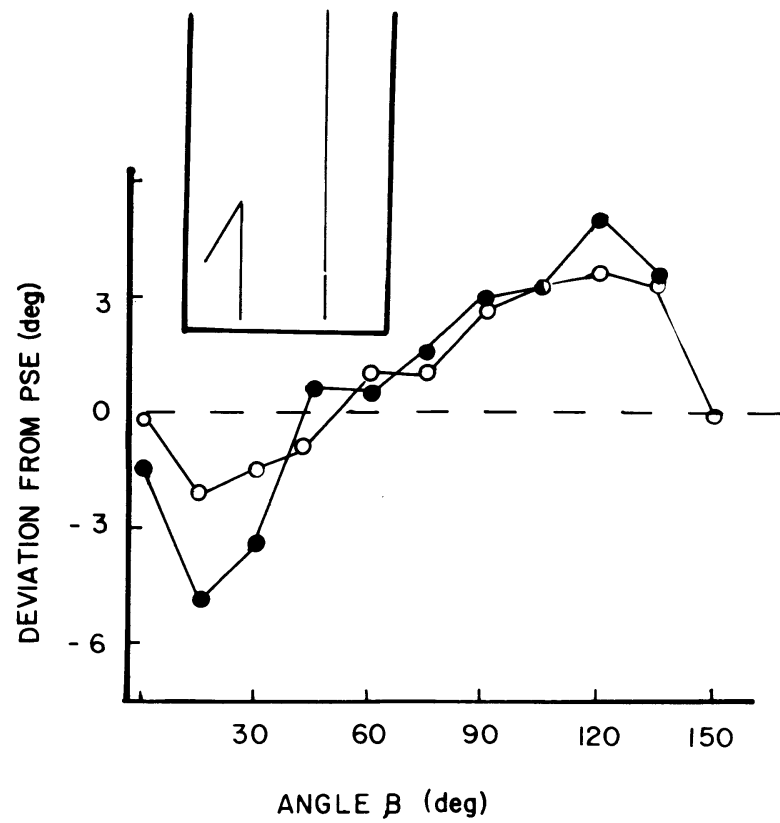

Figure 2. Size of illusion as a function of size of angle $\beta$ for angle $\alpha$ of $30 \mathrm{deg}$ (unfilled circles) and $45 \mathrm{deg}$ (filled circles). The dashed horizontal line indicates the PSE. Positive numbers on the ordinate indicate that an effect was obtained that was consistent in direction with the standard Poggendorff effect. Negative numbers indicate a reversed illusion. The average standard error of the mean was $1.18 \mathrm{deg}$ (ranging from $.78 \mathrm{deg}$ to $1.98 \mathrm{deg})$. There was no difference between the standard errors observed in the $\alpha=30 \mathrm{deg}$ and $\alpha=45 \mathrm{deg}$ conditions.

The observers had a separate response sheet for each stimulus. An example of the response figure is shown in the inset of Figure 2. Size of angle $\alpha$ in each response figure corresponded to that of the stimulus figure with which it was paired. The response sheets and the control figure were chosen on the basis of data indicating that the magnitude of the illusion is virtually zero for these configurations (Weintraub \& Krantz, 1971). The observers were instructed to judge the relation between the two transversal lines in the test figure and to place a slash on the right parallel of the response figure so that the perceived displacement of the transversal lines in the test figure was identical to the relation between the lower transversal and the slash on the response sheet. They were also informed that they should not use any extravisual aids and that they should refrain from using any pencil marks between the parallels of the response sheets. Half of the subjects judged the 30-deg $\alpha$ angle figures first, and this variable was counterbalanced with the random-order variable.

\section{Results}

The magnitude of the illusion for each stimulus figure was obtained by subtracting the mean height of the intersection of the response slash and the right parallel line from the mean height of the responses to the control figure. These data were then converted into perceived angular rotation of the transversal by a trigonometric transformation. Positive angular displacements represent clockwise rotation of the apparent orientation of the transversal, an effect consistent with the standard Poggendorff illusion. Negative displacements represent a counterclockwise rotation of perceived orientation of the transversal and a reversed illusion. 
As can be seen in Figure 2, manipulation of the size of angle $\beta$ had a large effect on magnitude of the Poggendorff illusion. The leftmost point of each function $(\beta=0)$ refers to stimulus figures with only $\alpha$ angles. A slight negative illusion was found for this condition, replicating a result found by Restle (1969). The rightmost point of each function shows the amount of illusion for the standard Poggendorff figures containing straight parallel lines. This configuration did not produce the largest effect on apparent orientation of the transversal. Rather, the largest illusion occurred when $\beta$ was $120 \mathrm{deg}$, regardless of the size of angle $\alpha$. For both sizes of $\alpha$, a reversed illusion obtained when angle $\beta$ was smaller than angle $\alpha$, and the strongest reversal occurred for a 15-deg $\beta$ angle. Thus, manipulation of angle $\beta$ systematically reversed and enhanced the standard Poggendorff illusion.

Although figures containing only $\alpha$ angles resulted in only a slight illusion, the size of $\alpha$ did interact with the effect of manipulating $\beta$ for figures containing both angles. Specifically, for figures with $\alpha=45 \mathrm{deg}$, varying $\beta$ resulted in a more pronounced Poggendorff effect than for $\alpha=30 \mathrm{deg}$. This is reflected in the modulation of illusion size (i.e., maximum Poggendorff illusion minus maximum Poggendorff reversal), which was $10.0 \mathrm{deg}$ for figures with $45-\mathrm{deg} \alpha$ angles and only $5.8 \mathrm{deg}$ for $30-\operatorname{deg} \alpha$ angles.

\section{Discussion}

Results of the present experiment indicate that independent manipulation of the size of angles $\alpha$ and $\beta$ by bending the parallel lines of the standard Poggendorff figure has large and systematic effects on the magnitude of the Poggendorff illusion. Furthermore, functions derived from an additive vector analysis of the Poggendorff figure using the angle-distortion data of Carpenter and Blakemore (1973) were fairly accurate in predicting the form of the empirical $\beta$ angle functions. However, independent manipulation of angle size unveils an unexpected interaction between the effects of these angles. This interaction was not predicted by the additive effects model. The model predicted the parallel functions, but the obtained effect of angle $\beta$ was larger when angle $\alpha$ was $45 \mathrm{deg}$ than when $\alpha=30 \mathrm{deg}$. This interaction is also reflected in the larger modulation in illusion magnitude found among stimuli with 45-deg $\alpha$ angles. There are, however, two variables confounded with size of angle $\alpha$ that might account for the observed interaction.

First, angle $\alpha$ size and orientation of the transverse line with respect to the page covaried in the present experiment. Previous data on the influence on the Poggendorff illusion of fixing the orientation of the transversal by rotating the entire figure has shown that the largest illusion obtains for a transversal oriented at $45 \mathrm{deg}$ (Leibowitz \& Toffey, 1966; Weintraub \& Krantz, 1971). The orientation effect is consistent with the angle-estimation data of Carpenter and Blakemore (1973), which show a reduction in the size of perceptual distortion of angle size as the orientation of the judged line departs from $45 \mathrm{deg}$ toward either the horizontal or the vertical meridian. The effect is also consistent with the effect of $\alpha$ found in the present experiment.

The second variable confounded with acute-angle size in Experiment 1 (and most other Poggendorff illusion studies that manipulate this variable) is distance between the apexes formed by the parallels and transversal segments. As the size of angle $\alpha$ decreases, the distance between the apexes (termed intertip distance, or ITD) increases if width between the parallels remains constant. Previous studies have shown that illusion magnitude, when measured by linear displacement of the transversal along the parallel increases linearly with distance between the parallels (Day \& Dickinson, 1976; Hotopf \& Olerearnshaw, 1972; Mackay \& Newbigging, 1977; Weintraub \& Krantz, 1971). This result is consistent with the notion that a perceptual rotation of transversal induces the Poggendorff illusion. Although this line of reasoning could not account for variation in the size of the illusion when measured in degrees, other unspecified effects of ITD may be responsible for the greater modulation of illusion magnitude as a function of angle $\beta$ size for the different $\alpha$ angles used in Experiment 1.

\section{EXPERIMENT 2}

In the first experiment, an unexpected interaction between the effects of angles $\alpha$ and $\beta$ was observed; that is, the effect of the size of angle $\alpha$ on the Poggendorff illusion was enhanced for figures with $\alpha=45 \mathrm{deg}$. In Experiment 2 orientation of the transversal segments and the distance between the inner tips of these segments were controlled to ascertain whether this interaction could be attributed to either of these confounding factors.

\section{Method}

Thirty-six undergraduates judged 54 test stimuli and 4 control stimuli. The 54 test stimuli consisted of 27 figures in which the width between the parallels was adjusted so that the ITD $=5.00 \mathrm{~cm}$ for all sizes of angle $\alpha$ and 27 figures in which the ITD was fixed at $8.66 \mathrm{~cm}$. Three $\alpha$ angles of 30,60 , and $90 \mathrm{deg}$ were combined with $\beta$ angles, ranging in size from $0 \mathrm{deg}$ to $180-\alpha \mathrm{deg}$, for a total of 27 figures. Half of the subjects judged rotated figures that were oriented so that the transversal lines were oriented at $45 \mathrm{deg}$ with respect to the page. The remaining subjects judged nonrotated figures that were similar to those used in Experiment 1, in that the orientation of the transversal varied with the size of angle $\alpha$. Individual subjects judged figures that were either rotated or nonrotated at both ITDs. Each observer in the nonrotated condition judged six control figures, three orientations at two ITDs. Only two control figures at a 45-deg orientation were judged by observers in the rotated condition. Stimulus construction, measurement of the illusion, and all other characteristics of the experimental design were similar to those used in Experiment 1.

\section{Results and Discussion}

Figure 3a shows the results for the nonrotated stimuli for ITD $=5.00 \mathrm{~cm}$. The results conform with those found in Experiment 1, in that there is an apparent interaction between size of angle $\alpha$ and the effects of angle $\beta$ on illusion magnitude. Rotating the stimulus figures so that the transversal was oriented at $45 \mathrm{deg}$ generally increased the magnitude of the illusion relative to the comparable nonrotated conditions. This effect is illustrated in Figure $3 \mathrm{~b}$.

The largest effect of rotation was on the $\alpha=90 \mathrm{deg}$ stimuli. The illusion was generally reversed for these figures, a result predicted by the additive angle misperception model, since a 90-deg angle is accurately perceived and acute angles are overestimated.

The curves for the various $\alpha$ angles in Figure 3b appear to be roughly parallel, suggesting an additive integration of the distortion of angles $\alpha$ and $\beta$. Thus, when ITD and orientation of the transversal are controlled, the additive vector model of the Poggendorff 


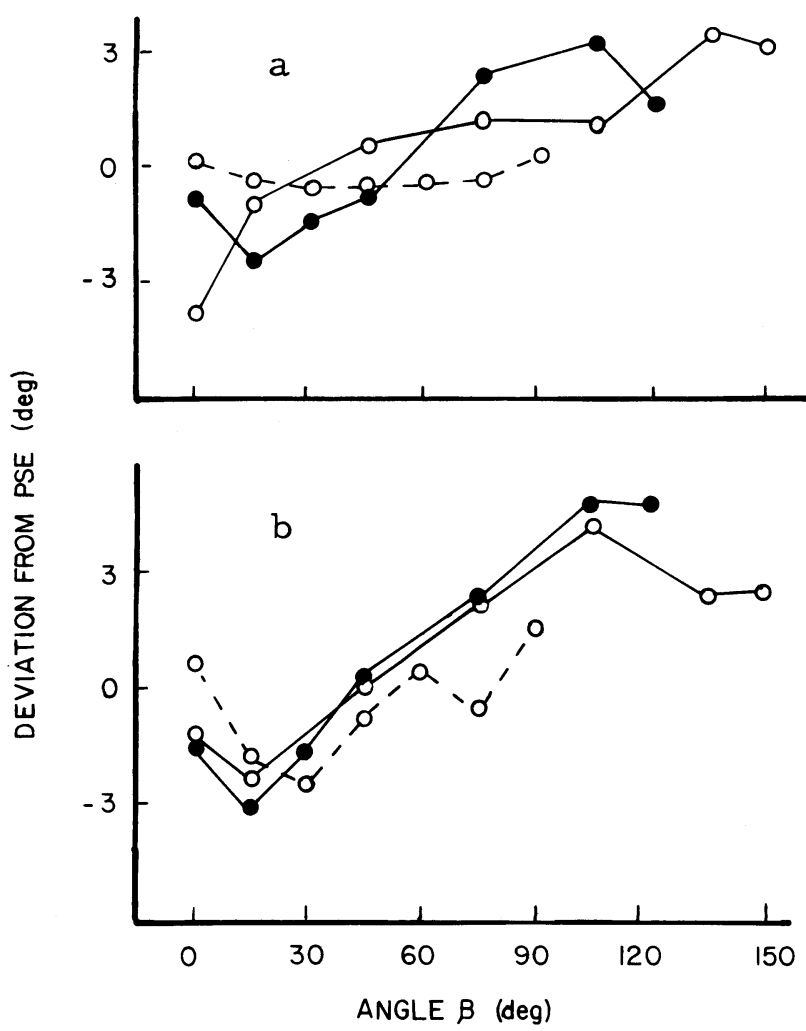

Figure 3. Results of Experiment 2 for stimulus figures with ITD $=5.00 \mathrm{~cm}$. Results for nonrotated figures are shown in Figure 3a, and Figure 3b shows results from rotated stimuli. Each curve represents illusion magnitude as a function of angle $\beta$ size for one size of angle $\alpha$. For unfilled circles/solid lines, $\alpha=30 \mathrm{deg}$; filled circles, $\alpha=60 \mathrm{deg}$; unfilled circles/broken lines, $\alpha=90 \mathrm{deg}$. The average standard error of the mean was $1.35 \mathrm{deg}$ (ranging from 48 to $2.70 \mathrm{deg}$ ) for all conditions except the 90-deg nonrotated condition. In this condition, the standard error was smaller (mean $=.35$, ranging from .18 to $.59 \mathrm{deg}$ ), a result that reflects the accuracy of perception of horizontal meridian.

illusion predicts the effects of bending the parallels fairly well. Data obtained for the stimuli with an ITD of $8.66 \mathrm{~cm}$ showed a pattern of results that were quite similar to those found with an ITD of $5.00 \mathrm{~cm}$, although absolute illusion magnitude was generally smaller for the larger ITD.

\section{GENERAL DISCUSSION}

The present study investigated the independent contributions of acute and obtuse angles to the Poggendorff illusion. The results of the first experiment showed that, by manipulating angle $\beta$ size, the illusion could be enhanced or reversed in accordance with the predictions made from Carpenter and Blakemore's (1973) angle-distortion data. However, an unpredicted interaction between the effects of angle $\alpha$ and angle $\beta$ on the illusion was also found. The second experiment showed that when orientation of the transversal line and ITD were controlled, this interaction was eliminated, suggesting an additive combination of the effects of the size of angles $\alpha$ and $\beta$. Thus, effects of angle $\alpha$, angle $\beta$, orientation of the transversal, and ITD were isolated in the present study.

Although other factors (e.g., the horizontal-vertical effect, perceptual contraction of the distance between the parallels; see Day \& Dickinson, 1976) can affect the size of the observed illusion, experiments of the present study show that angle size is a major determinant of the size of the effect that is obtained. Furthermore, our results suggest that the illusion is, to a large extent, a result of the misperception of the orientation of the transversal caused by perceptual distortion of angle size. However, the present experiments show only indirectly that angle size and illusion magnitude are related. They do not show that the angles of the Poggendorff figure are misperceived. Although angle-distortion data can be used to predict the effects of angle size fairly closely, attempts to directly validate the role of angle misperception of the Poggendorff illusion have been unsuccessful (e.g., Hotopf \& Ollerearnshaw, 1972). The failure to find an adequate distortion of perceived orientation of the transversal may be due to the use of insensitive measures. Alternatively, it may be that transversal orientation is not distorted, but that angle size is somehow associated with some other process that results in the Poggendorff illusion. If angle mispreception is indeed responsible for the effects reported here, then a lateral inhibitory neural displacement model such as that proposed by Carpenter and Blakemore (1973) could account for them.

\section{REFERENCES}

Bouma, H., \& Andriessen, J. J. Induced changes in perceived orientation of line segments. Vision Research, 1970, 10, 333-349.

Carpenter, R. H. S., \& Blakemore, C. Interactions between orientations in human vision. Experimental Brain Research, 1973, 18, 287-303.

Day, R. H., \& Dickinson, R. G. The Poggendorff illusion: Misalignment which is not attributable to apparent orientation of the transversals. Quarterly Journal of Experimental Psychology, 1975, 27, 551-557.

Day, R. H., \& Dickinson, R. G. The components of the Poggendorff illusion. British Journal of Psychology, 1976, 67, 537-552.

Hotopf, W. H. N., \& Ollerearnshaw, C. The regression to right angles tendency and the Poggendorff illusion II. British Journal of Psychology, 1972, 63, 369-379.

Leibowitz, H., \& Toffey, S. The effects of rotation and tilt on the magnitude of the Poggendorff illusion. Vision Research, 1966, 6, 101-103.

Mackay, D. C., \& Newbigging, P. L. The Poggendorff and its variants do arouse the same perceptual process. Perception \& Psychophysics, 1977, 21, 26-32.

Pressey, A. W., \& Sweeney, O. Acute angles and the Poggendorff illusion. Quarterly Journal of Experimental Psychology, 1972, 24, 164-174.

Restle, F. Illusions of bent line. Perception \& Psychophysics, 1969, 5, 273-274.

Velinsky, S. Explication physiologique de l'illusion de Poggendorff. Année Psychologique, 1925, 26, 107-116.

Weintraub, D. J., \& Krantz, D. H. The Poggendorff illusion: Amputations, rotations, and other perturbations. Perception \& Psychophysics, 1971, 10, 257-264.

(Received for publication April 1, 1980.) 